\title{
Recuperación de tejidos blandos de perezosos fósiles gigantes (Mammalia, Xenarthra, Pilosa) de la zona central del Departamento de Yoro, Honduras
}

Soft tissue recovery from giant fossil sloths (Mammalia, Xenarthra, Pilosa) of the central zone of the Department of Yoro, Honduras

DOI 10.5377/rct.v0i24.7875

Leonel E. Zúniga ${ }^{1}$

Lourdes Enríquez ${ }^{2}$

Carlos López-Videz ${ }^{3}$

David Aguilar ${ }^{4}$

\section{RESUMEN}

El estudio microestructural de los fósiles ha crecido significativamente en las últimas dos décadas. La recuperación de tejidos blandos y fragmentos de moléculas a partir de la desmineralización de huesos fósiles ha sido reportada por varios autores abriendo nuevas rutas en el estudio de los fósiles. El propósito del presente estudio fue explorar la recuperación de tejidos blandos de restos óseos fósiles de perezosos gigantes recientemente colectados en la zona central del Departamento de Yoro, Honduras. Los fragmentos de hueso esponjoso fueron desmineralizados utilizando EDTA, de los cuales se recuperaron restos de un aparente vaso sanguíneo, además se observaron al microscopio osteocitos y probables restos de glóbulos rojos. Los resultados evidencian tejidos blandos del tejido óseo esponjoso, preservados en medio de las condiciones ambientales que indujeron la fosilización de los huesos, representando una fuente potencial para futuros estudios celulares y moleculares, así como preguntas sobre su conservación a través del tiempo.

Palabras clave: Mamíferos, tejidos blandos, vasos sanguíneos, osteocitos, glóbulos rojos, hueso esponjoso, preservación.

1 Profesor del Departamento de Biología, Escuela de Biología, Facultad de Ciencias, UNAH: leonel.zuniga@unah.edu.hn

2 Profesora del Instituto de Investigaciones en Microbiología, Facultad de Ciencias,UNAH: lourdes@unah.edu.hn

${ }^{3}$ Escuela de Microbiología, Facultad de Ciencias, UNAH: carlos.vides@unah.edu.hn

${ }^{4}$ Profesor de la Escuela de Física, Facultad de Ciencias, Coordinador del Laboratorio de Microscopía Electrónica, UNAH: david.aguilar@unah.edu.hn 


\section{ABSTRACT}

The microstructural study of fossils has grown significantly in the last two decades. The recovery of soft tissues and fragments of molecules from the demineralization of fossil bones has been reported by several authors opening new routes in the study of fossils. The purpose of this study was to explore the recovery of soft tissues of fossil skeletal remains of giant sloths recently collected in the central area of the Department of Yoro, Honduras. Fragments of cancellous bone were demineralized using EDTA, from which remains of an apparent blood vessel were recovered, and in addition, osteocytes and probable remains of red blood cells were observed under the microscope. The results show soft tissues of the cancellous bone tissue, preserved in the middle of the environmental conditions that induced the fossilization of the bones, representing a potential source for future cellular and molecular studies, as well as questions about its conservation through time.

Keywords: Mammals, osteocytes, soft tissues, red blood cells, blood vessels, cancellous bone, preservation. 


\section{INTRODUCCIÓN}

El registro fósil ha evidenciado una enorme capacidad de preservación de diversos tipos de estructuras de los seres vivos. Como ejemplo de lo anterior se incluyen la preservación de plumas (Qiang, Currie, Norell, y Shu-An, 1998; Schweitzer et al., 2002; Xu, Wang, y Wu, 1999), cabello (Franzen, 2017), tejido blando embrionario (Chiappe et al., 1998), tejido muscular y órganos internos (Briggs, Wilby, Pérez-Moreno, Sanz, y Fregenal-Martínez, 1997; Kellner, 1996; Sasso y Signore, 1998), piel (Manning et al., 2009), y estructuras celulares (Pawlicki, Korbel, y Kubiak, 1966). Se suma a lo anterior los casos de preservación de tejidos momificados de organismos en ámbar (Grimaldi, Bonwich, Delannoy, y Doberstein, 1994).

El artículo publicado por Schweitzer et al (2005) reportando la recuperación de tejidos blandos de Tyrannosaurus rex, evidenciando microestructuras residuales propias de los huesos, fue catalogado como "controversial" (Pappas, 2013). Lo anterior debido al concepto clásico de fosilización y también por las implicaciones en el entendimiento de los procesos que favorecen la degradación de los tejidos. Ejemplos de estos procesos son la autólisis, heterotrofia bacteriana aerobia y anaeróbica, carroñeo y bioturbación (Xiao y Knoll, 1999). En consecuencia, la preservación de tejidos blandos despierta importantes interrogantes sobre los mecanismos de preservación durante largos periodos de tiempo, las tasas de degradación de los diferentes tipos de compuestos orgánicos y en última instancia los métodos de datación de un fósil.

Resalta en la historia del estudio de la microestructura de los fósiles Reinhold Fritz-Gaertner, geólogo de orígen prusiano quien perteneció al Museo de Historia Natural del Estado de New York, EEUU (New York State Museum of Natural History, 1878) y que realizó algunos de los primeros estudios minerológicos, geológicos y paleontológicos de Honduras (Sapper, 1905), recomendando a finales del siglo XIX la importancia de incluir el estudio microscópico en el estudio de los fósiles (Fritz-Gaertner, 1878). En el siglo XX, Gross (1934) y Enlow y Brown (1956) realizaron importantes estudios microestructurales de fósiles. Los estudios de Donald Enlow contribuyeron significativamente a la paleohistología e histología ósea comparada (de Ricqlès, 2007).

La recuperación de restos de tejidos blandos a partir de huesos fósiles fue comentada posteriormente por Pawlicki (1978) y Pawlicki, Korbel y Kubiak (1966). A partir de Schweitzer et al (2005) otros estudios han fortalecido el tema, implicando también la recuperación de fragmentos de proteínas (Armitage y Anderson, 2013; Bertazzo et 
al., 2015; Cadena, 2016; Cadena y Schweitzer, 2012; Cleland, Schroeter, Feranec, y Vashishth, 2016; Cleland et al., 2015; O'Reilly, Summons, Mayr, y Vinther, 2017; Plet et al., 2017; Schweitzer, Wittmeyer, y Horner, 2007; Schweitzer, Zheng, Cleland, y Bern, 2013; Schweitzer et al., 2009). Buckley et al (2017) cuestionaron la recuperación de secuencias peptídicas de fósiles de dinosaurio aduciendo una posible contaminación cruzada. Sin embargo, Schroeter et al (2017) demostraron que las secuencias peptídicas pueden obtenerse de manera confiable y reproducible a partir de fósiles y que las secuencias fragmentarias para proteínas antiguas pueden expandirse aun más.

Por otro lado, la identificación de factores que podrian favorecer la preservación de tejidos blandos en fósiles ya habia sido abordado por Allison (1988) y Briggs (1991), proponiendo como factores: las diferencias entre los tipos de tejidos ante los procesos de degradación, sistemas de deposición particulares, el momento en el que inicia la mineralización despues de la muerte de los organismos y las condiciones ecologicas propias de esos organismos. Schweitzer et al (2014), han sugerido la participacion del hierro en la preservacion de los tejidos blandos. Dentro de este contexto también se ha sugerido para algunos casos de preservación en fósiles la participacion microbiana (Briggs y McMahon, 2016), aunque como lo expresan Schweitzer, Moyer, y Zheng (2016), los microorganismos difícilmente pueden reproducir la morfología característica de celulas de gran tamaño como los osteocitos.

El registro fósil de elementos óseos mineralizados de megafauna mamífera es abundante, pero muy pocos estudios se han realizado buscando recuperar restos de tejidos blandos propios de estos elementos anatómicos. En cuanto a la recuperación de tejidos blandos y fragmentos de moléculas biológicas a partir de huesos fósiles de mamíferos resalta la recuperación de elementos celulares de fósiles de mastodonte y mamut (Schweitzer et al., 2007), recuperación de elementos celulares de fósiles de pangolín (Cadena, 2016); y recuperación de fragmentos de proteínas de Castor gigante (Cleland et al., 2016).

La recuperación de tejidos blandos de elementos fósiles y posteriormente fragmentos de proteínas, ha desencadenado el inicio de una nueva rama de la ciencia denominada "paleoproteómica". La Paleoproteómica es un subcampo de rápido crecimiento de la paleontología molecular (Asara, Schweitzer, Freimark, Phillips, y Cantley, 2007; Cleland et al., 2016; Cleland et al., 2015; Organ et al., 2008; Schweitzer et al., 2009).

En cuanto al registro paleontológico, Honduras es el primer país de Centroamérica del que se reportó el descubrimiento de fósiles de vertebrados (Lucas, Bonta, 
Rogers, y Alvarado, 2011). Honduras cuenta con un significativo número de sitios reportados de fósiles de vertebrados (Fernández, 1983) y cuenta con el único reporte publicado a la fecha de fósil de dinosaurio de Centroamérica (Horne, 1994; Lucas, 2014; Lucas y Alvarado, 2017; Weishampel, Dodson, y Osmólska, 2004; Zúniga, 2017a).

El registro fósil de perezosos terrestres gigantes en Centroamérica y de otros mamíferos extintos ha sido considerado una de las bases más importantes para la teoría del Gran Intercambio Biológico Americano. La teoría del Gran Intercambio Biológico Americano es considerada como la evidencia más importante para el fechado del cierre de Centroamérica y la conexión entre Norteamérica y Suramérica (Molnar, 2008; Osborn, 1910), sin embargo esta temática se encuentra actualmente bajo un intenso debate (Erkens y Hoorn, 2016; Jaramillo et al., 2017; Molnar, 2017; Montes et al., 2015; Stone, 2013; Zúniga, 2017a) y el registro fósil de dinosaurio de Honduras (Horne, 1994) representa un aspecto sobresaliente en esta discusión.

En cuanto a la antigüedad de fósiles de perezosos terrestres gigantes de Honduras, Webb y Perrigo (1984) consideran los fósiles reportados del sitio "Orillas del Rio Humuya" en el Valle de Comayagua (Megalonyx sp, Eremotherium sp), como "Pleistoceno tardío", lo que también es reafirmado por Cartelle y De luliis (1995), es decir 126,000 a 11,000 años de antigüedad. Los estudios de Rincón (2004), Gallo, Avilla, Pereira, y Absolon (2013), y Varela, Tambusso, Patiño, Di Giacomo, y Fariña (2017), evidencian una distribución de perezosos terrestres gigantes como Eremotherium sp a través de Centroamérica.

El propósito de este estudio fue explorar la preservación de tejidos blandos de restos óseos de perezosos terrestres gigantes recientemente colectados en Honduras. Este estudio contribuirá a impulsar en Centroamérica el estudio paleontológico de las microestructuras presentes en fósiles y brindará información comparativa con los resultados obtenidos por otros estudios.

\section{MÉTODOS}

Sitio de Colecta

Con el fin de colectar y estudiar posteriormente la macro y microestructura de fósiles, la Facultad de Ciencias de la UNAH organizó en junio del año 2017 una gira de 
campo al Departamento de Yoro a un sitio reportado a la Escuela de Biología, al noroeste de la ciudad de Yoro, al suroeste de la cuenca hidrográfica del rio Aguán (Figura 1).

Figura 1. Sitio de colecta de los fósiles, zona central del Departamento de Yoro, Honduras

Fuente: Leonel Zúniga

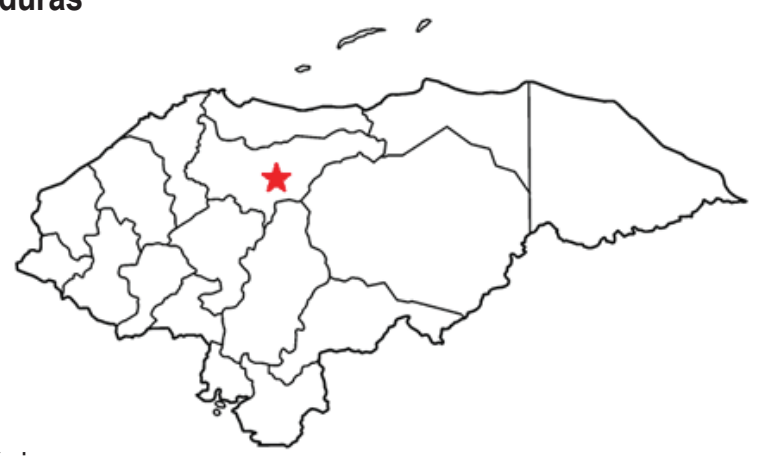

Material colectado

La gira tuvo como resultado identificar un afloramiento con fósiles (Figura 2) con características de tafocenosis, y la colecta de 69 ejemplares, entre fragmentos y huesos completos (Zúniga, 2017b), de los cuales la mayoría pertenecen a mamíferos del superorden Xenarthra, orden Pilosa, suborden Folivora, (perezosos gigantes) y dos ejemplares fósiles que pertenecen al grupo de los équidos. Desde el punto de vista geológico, el afloramiento, cuya orientación general es de $\mathrm{N}-\mathrm{E}$, en su mayoría está compuesto por areniscas de color café claro con tamaño de grano pequeño y mezcla de arrastre de rocas de la Formación Gracias y Padre Miguel (IHCIT, 2017). Los fósiles recuperados fueron agregados a la colección de fósiles de la UNAH en Ciudad Universitaria, de los cuales se tomaron muestras para realizar el presente estudio.

Se realizó un ensayo preliminar un mes después de la extracción y colecta de los fósiles y dos ensayos adicionales seis y ocho meses después de la colecta. En el ensayo realizado a los seis meses de la colecta, se tomó dos muestras a partir de los fósiles colectados en el sitio descrito : Muestra A) (Objeto 0060) fragmentos (equivalente a $1 \mathrm{~cm} 3$ ) de hueso esponjoso de la apófisis espinosa de una vértebra dorsal (Figura 3), Muestra B) (Objeto 0004) Fragmentos $(3 \mathrm{~cm} \times 1.5 \mathrm{~cm} \times 1 \mathrm{~cm})$ de hueso esponjoso de fémur con estructura canalicular presente de 2.5 milímetros de diámetro y $3 \mathrm{~cm}$ de largo (Figura 4), extraída de una región cercana a un foramen nutricio del hueso. 
Figura 2. Fragmento de fémur de perezoso gigante. Fotografía in situ previo a la etapa de colecta, noroeste de Yoro, Departamento de Yoro, Honduras.

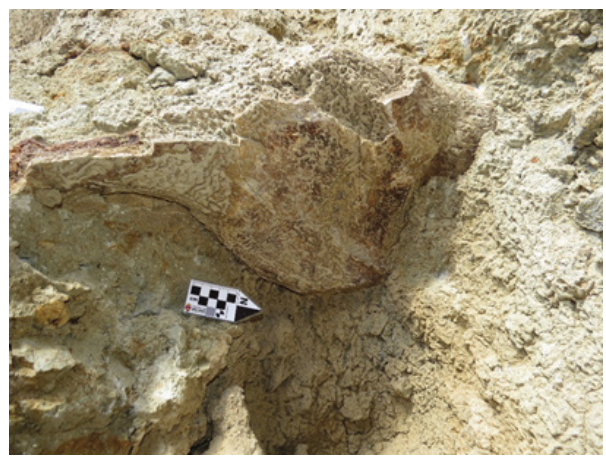

Fuente: Elvin Hernández, gira de campo 2017.

Figura 3. Colecta de fragmentos de hueso esponjoso de apófisis espinosa de vértebra dorsal (Muestra $\mathrm{A}$ ).

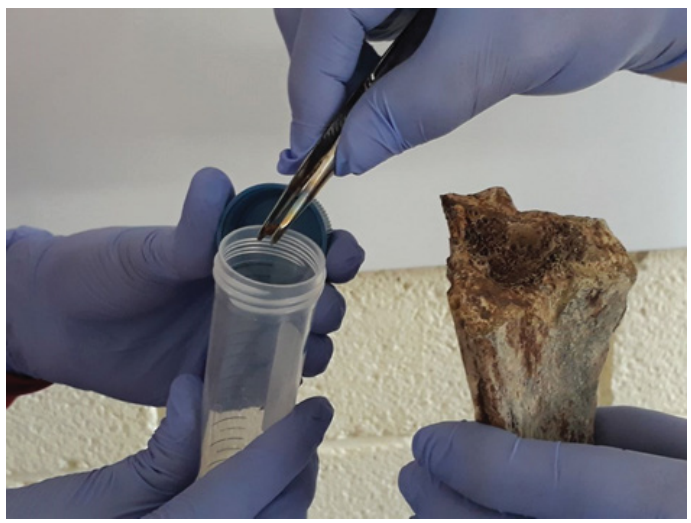

Fuente: Leonel Zúniga

Figura 4. Fragmentos de hueso esponjoso de fémur con estructura canicular presente (Muestra B).

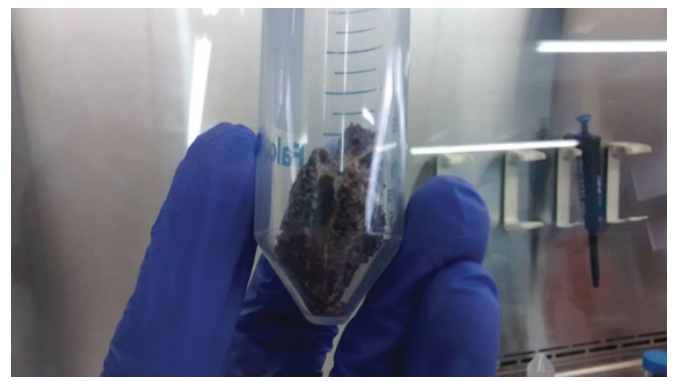

Fuente: Leonel Zúniga 
Para la desmineralización se tomó en cuenta la metodología provista por Schweitzer et al (2005). Los tejidos se desmineralizaron utilizando $\operatorname{EDTA}(0,5 \mathrm{M}, \mathrm{pH} 8,0)$ durante 7 días, cambiando el tampón diariamente. El ensayo preliminar un mes después de la extracción de los fósiles se realizó con cantidades diferentes de EDTA por muestra $(4 \mathrm{ml}$ y $6 \mathrm{ml})$ para seleccionar el volumen más favorable. El último día, después de retirar el EDTA, el material restante de la muestra fue lavado con tampón de fosfato (PBS, pH 6,8). Inmediatamente después de agregar el tampón de fosfato se tomó muestras de las diferentes fases visibles en el tubo de ensayo, y se observó al microscopio óptico.

En el ensayo realizado 6 meses después de la colecta, la muestra A se sumergió en $4 \mathrm{ml}$ de EDTA durante siete días, mientras que la muestra $B$ se roció superficialmente con EDTA durante tres días, y se sumergió en $10 \mathrm{ml}$ de EDTA durante 4 días adicionales. El tampón retirado de la muestra $A$ de los últimos tres días se acumuló en un recipiente adicional, mostrando material sedimentado. El último día, después de retirar el EDTA, el material restante de las muestras A y B fue lavado con tampón de fosfato (PBS, pH 6,8). Inmediatamente después de agregar el tampón de fosfato se tomó muestras de la fase intermedia del sedimento de la muestra A y se observó al microscopio óptico. También se tomó muestras del material sedimentado de los recipientes con EDTA retirado de la muestra $A$ y acumulado los últimos 3 días, todos los sedimentos se observaron al microscopio. Se utilizó un microscopio Olympus CX31 en ampliación de 40x con cámara DP21, el software DP2-Twain para tomar las fotomicrografías y se utilizó Software Axiovision para el procesamiento digital de las mismas.

Una semana después de este procedimiento se inició la fijación del material sedimentado de los recipientes con EDTA retirado de la muestra $A$, utilizando solución de formalina durante 6 horas, y se aplicó una cadena de deshidratación con etanol durante 24 horas iniciando con una concentración al 30\% y finalizando con una concentración al 95\%, centrifugando antes de cada aplicación. Se aplicó dos cambios de Tert-butanol durante 18 horas. Al finalizar este proceso se inició la etapa de desecación en frio y al vacío de una pequeña muestra adaptando la metodología de Inoué y Osatake (1988). Después de 1 hora de desecación se aplicó un baño de oro-paladio a la muestra mediante un cobertor iónico. El preparado se observó en un microscopio electrónico de barrido, Hitachi S-2700. La Muestra B se observó en fresco utilizando un microscopio digital de superficie Dino-Lite y se utilizó el software Dino-capture para la generación de fotografías y video. 


\section{RESULTADOS}

El ensayo preliminar permitió valorar el efecto de la exposición a cantidades diferentes de EDTA. La muestra de 1cc de tejido óseo esponjoso expuesta a 4ml de EDTA en el ensayo preliminar mostró una mejor recuperación de estructuras celulares que la muestra expuesta a $6 \mathrm{ml}$.

En el ensayo preliminar se observó estructuras similares a células características de la estructura del tejido óseo, como osteocitos (con filopodios y potencial preservación de contenido intracelular), así como estructuras de apariencia vascular, estructuras esféricas y semiesféricas y aparentes cúmulos celulares. (Figura 5).

En el ensayo final se recuperaron nuevamente osteocitos y estructuras de apariencia vascular a partir de la muestra A (Figura 6 y 7). En la observación al microscopio electrónico de barrido se identificó estructuras que son en efecto osteocitos con sus filopodios (Figura 8), con dimensiones similares a las observadas al microscopio óptico. La desmineralización parcial de la muestra B permitió observar restos de tejido blando de una aparente estructura vascular (vaso sanguíneo) con derivación a otra de menor diámetro (Figura 9).

Figura 5. La desmineralización de tejido óseo esponjoso (perezoso terrestre gigante, ensayo preliminar) presenta estructuras de tejido blando: a) Osteocito de perezoso gigante, b) Estructuras de forma vascular observado, c) Cúmulo de estructuras esféricas y semiesféricas, d) Estructuras esféricas o semiesféricas contraídas dentro de aparente estructura vascular, e) Estructura esférica, f) Osteocito de perezoso gigante
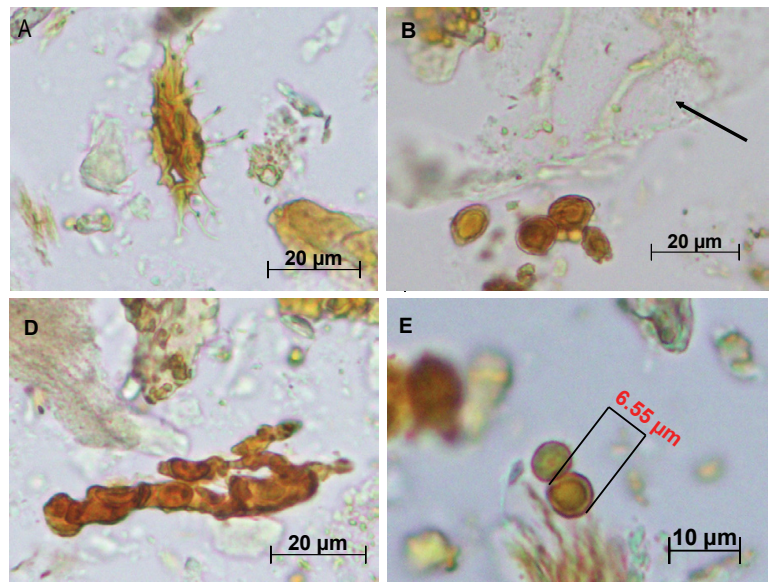

Fuente: Leonel Zúniga, Carlos Vides.

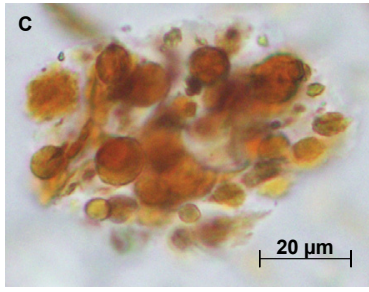

$F$

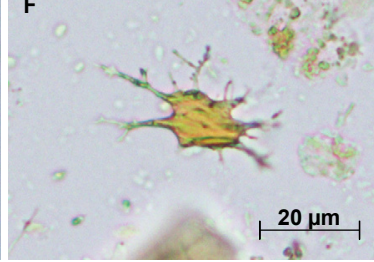


Figura 6. La desmineralización de tejido óseo esponjoso (perezoso terrestre gigante, ensayo final, Muestra A, evidencia estructura de apariencia vascular, con estructuras globulares de color rojizo en su interior

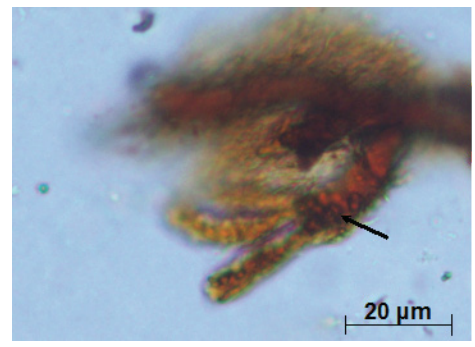

Fuente: Leonel Zúniga, Carlos Vides.

Figura 7. La desmineralización de tejido óseo esponjoso (perezoso terrestre gigante, ensayo final, Muestra A, presenta estructuras de tejido blando a), b), c), y d) Osteocitos con presencia de filopodios.
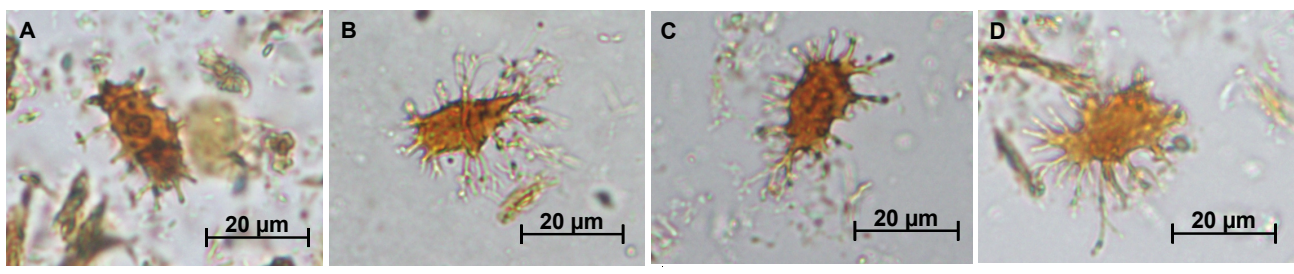

Fuente: Leonel Zúniga, Carlos Vides.

Figura 8. La desmineralización de tejido óseo esponjoso (perezoso terrestre gigante; ensayo final, Muestra A, presenta estructuras (a y b) que aparentan ser osteocitos con filopodios.(Microscopio Electrónico de Barrido Hitachi S-2700, voltaje de aceleración $6 \mathrm{kV}$, muestras preparadas mediante desecación en frio con alcohol T-Butil, y recubiertas con aleación de oro-paladio mediante un cobertor iónico)
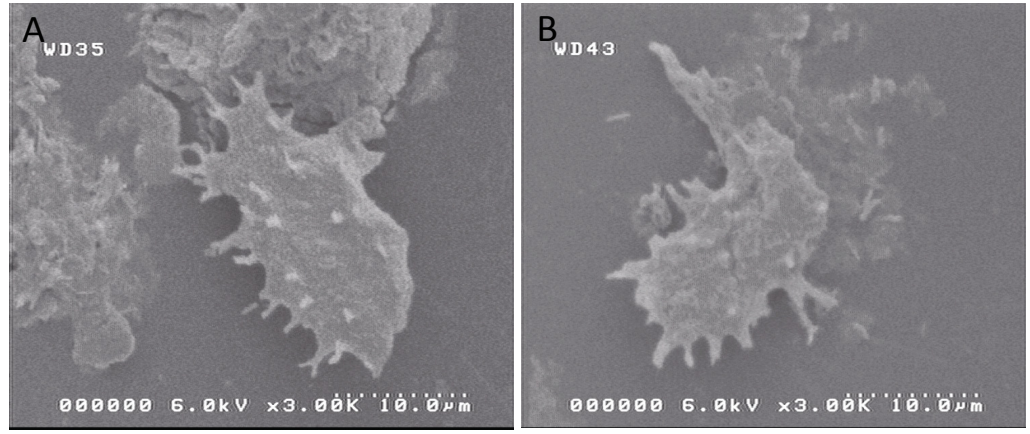

Fuente: David Aguilar. 
Figura 9. La desmineralización parcial de fragmentos de hueso esponjoso (perezoso terrestre gigante, ensayo final, Muestra B), presenta estructura de tejido blando: a) Estructura canalicular de tejido blando continuo de $2.5 \mathrm{~mm}$ de diámetro y $3 \mathrm{~cm}$ de largo, b) Derivación de la estructura canalicular en ángulo de 33.8 grados a un conducto de menor diámetro
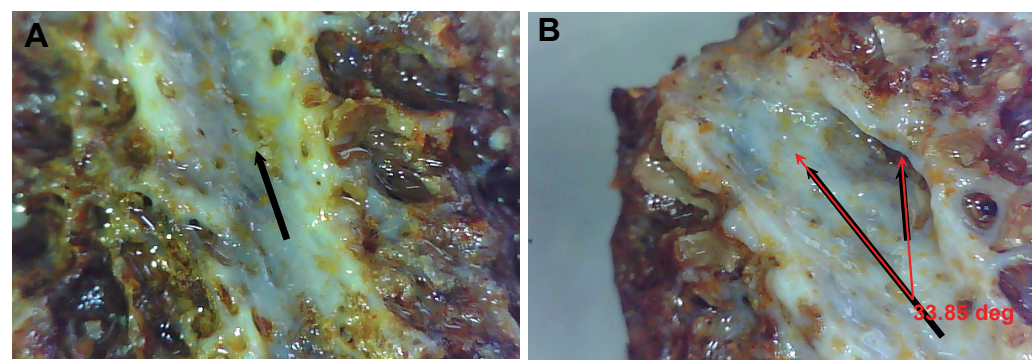

Fuente: Leonel Zúniga

\section{DISCUSION}

En las muestras observadas al microscopio tanto del ensayo preliminar como del ensayo final se evidencian osteocitos que retienen filopodios así como estructuras vasculares conteniendo en su interior estructuras de forma globular y color rojo que podrían representar glóbulos rojos o restos de ellos. Estos resultados son consecuentes con los datos descritos por Schweitzer et al (2007), en cuanto a la recuperación de osteocitos y vasos a partir de tejido óseo de fósiles de mamut (Mammuthus columbi) y osteocitos a partir de tejido óseo de fósiles de mastodonte (Mammut americanum). Cadena (2016) reporta también resultados similares al desmineralizar tejido óseo de fósiles de pangolín extinto (Eomanis krebsi) recuperando osteocitos.

La observación con un microscopio superficial y la manipulación de la estructura de forma canalicular con una pinza metálica estéril, permitió evidenciar la textura suave y flexible del tejido, lo que aparenta ser restos de un vaso sanguíneo y su bifurcación macroscópicamente visibles. El procedimiento de desmineralización parcial inicial aplicado a la muestra $B$ resultó efectivo para recuperar estructuras macroscópicas. En cuanto a la presencia de restos de tejido vascular en fósiles, los resultados obtenidos, evidenciando estructuras macro y microscópicas asociadas (como posibles eritrocitos); Schweitzer et al (2007) reporta resultados similares al recuperar restos aparentes de vasos y células sanguíneas de fósiles de dinosaurio (Tyrannosaurus rex). Evidencias solidas de preservación de glóbulos rojos han sido documentadas al menos en huesos humanos de 2000 años de antigüedad (Maat, 1993) y en restos humanos momificados de 5000 años de antigüedad (Janko, Stark, y Zink, 2012). 
Briggs (1991), propone que los tejidos blandos de un organismo solo pueden sobrevivir cuando se inhibe la descomposición y la mineralización es muy rápida. Para que lo anterior ocurra, los cadáveres deben ser depositados rápidamente en un ambiente sedimentario que promueva la mineralización diagenética rápida. Alisson, (1988), describe la relación entre la preservación de tejidos blandos y una rápida mineralización, (Figura 10).

Figura 10. Las tasas relativas de descomposición y mineralización determinan el grado en que muchos de los tejidos blandos se conservan. Este diagrama conceptual muestra la relación entre la descomposición y la mineralización en la preservación de varios elementos de una biota.

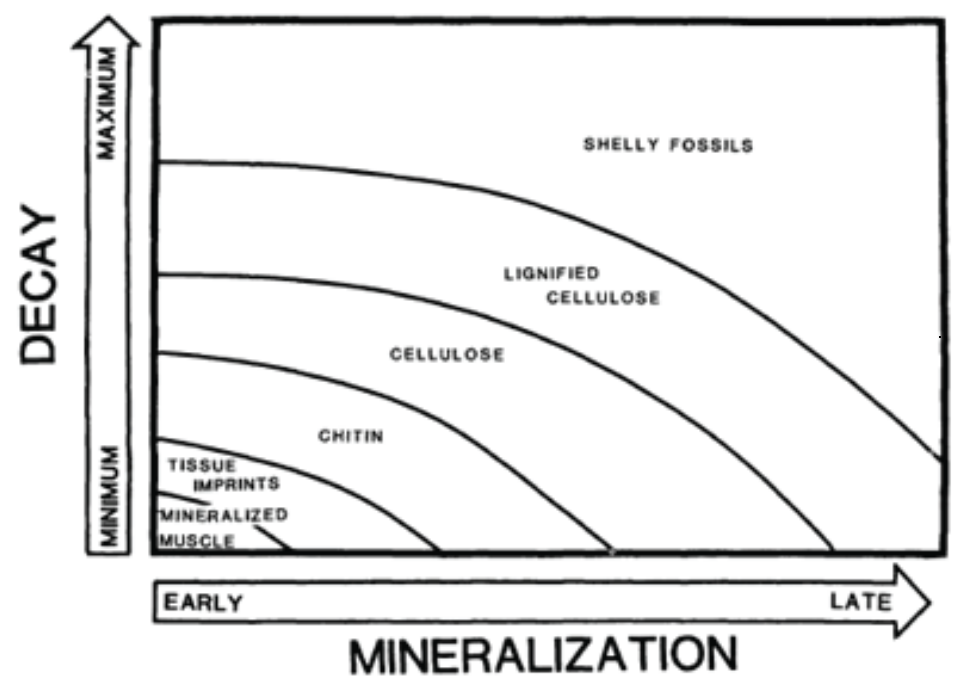

Fuente: : Allison, 1988

Hare (1988), plantea que el grado de preservación de un hueso en un ambiente natural después de la muerte del animal es una función del tiempo y del ambiente, y su sobrevivencia en el ambiente depende también de la fortaleza mecánica y dureza del hueso. En el caso de los fósiles, el estado de preservación puede brindar información del ambiente diagenético (Hare, 1988).

La recuperación de restos de tejido blandos propios del hueso esponjoso de forma confiable y reproducible a partir de fósiles, plantea preguntas sobre la potencial recuperación de otros tejidos propios de los huesos de mamíferos, y la necesidad de impulsar este tipo de investigaciones en fósiles colectados en Honduras y Centroamérica. 


\section{CONCLUSIONES}

Porciones de tejidos blandos del tejido óseo esponjoso han sido preservados en medio de las condiciones ambientales que indujeron la fosilización de los huesos. Se identifican osteocitos conservados, así como probables restos de vasos sanguíneos, que pueden representar una fuente potencial de información de datos celulares y moleculares de estas especies.

Los resultados confirman que la metodología aplicada en este y otros estudios para desmineralizar huesos fósiles permite recuperar tejidos blandos que formaron parte de la estructura original de estos elementos anatómicos. Estos resultados refuerzan lo concluido por otros autores respecto a la necesidad de revisar los modelos de los procesos de fosilización.

Al recuperar tejidos blandos de fósiles se abren nuevos campos de estudio, requiriendo un perfeccionamiento de las técnicas de desmineralización, desarrollar nuevos métodos de datación mediante el estudio de estos restos celulares y las sustancias absorbidas por ellos, y en general la elaboración de perfiles de los fósiles que tomen en cuenta los residuos de las moléculas de la composición química original.

\section{AGRADECIMIENTOS}

Agradecemos al Instituto de Investigaciones en Microbiología de la Escuela de Microbiología de la UNAH, al Centro de Innovación en Tecnologías de Información y Comunicación para Ciencias Biológicas y al Laboratorio de Microscopía Electrónica de la Escuela de Física de la UNAH por haber hecho posible el desarrollo de esta investigación; Nabil Kawas Decano de la Facultad de Ciencias, María Luisa Niño Asistente Estratégico de la Decanatura de la Facultad de Ciencias, Sara Serpas Gestor Administrativo de la Facultad de Ciencias, Elia Marta Sarmiento Directora Escuela de Biología, Alicia Cárcamo Jefa del Departamento de Biología, Instituto Hondureño de Ciencias de la Tierra, Gerardo Moreira Director de Servicios Generales de la UNAH, Omar Talavera del Instituto Hondureño de Antropología e Historia y las Fuerzas Armadas de Honduras por su apoyo financiero y logístico para la realización de la gira de colecta de los fósiles en junio 2017; Dirección Nacional de Patrimonio Cultural y el Museo de Historia Natural de El Salvador por la asistencia técnica recibida durante el desarrollo de la gira; Elvin Hernández, Ana Natarén, Cesar Cruz, Santos Montoya, 
Pablo Ochoa, Gerson Salas y Herman Álvarez, así como a Eric Matute y Dennis Cabrera, que fueron parte directa o indirectamente de la gira de campo; Irasema Montoya, Gerardo Borjas, Audato Paz, German Sandoval, Lilian Ferrufino, Luis López, Moisés Sánchez, Mireya Zelaya y al Museo de Historia Natural de la Escuela de Biología, Jorge Carrasco de la Escuela de Microbiología de la UNAH y Franklin Castañeda por el apoyo, asesoramiento y colaboración recibido durante esta investigación.

También nuestro agradecimiento a Nereyda Estrada por el asesoramiento durante la preparación y desarrollo de la gira de campo, la investigación y la revisión de este artículo, así como a Edwin Cadena por la revisión en las etapas iniciales de preparación de este artículo y sus importantes recomendaciones.

Expresamos también nuestra gratitud a Nahín George y su familia y Darlin Leal por su apoyo entusiasta y asistencia logística antes y durante la gira de campo realizada en Yoro.

\section{REFERENCIAS BIBLIOGRÁFICAS}

Allison, P. A. (1988). Konservat-Lagerstatten: Cause and Classification. Paleobiology, 14(4), 331-344. Armitage, M. H., y Anderson, K. L. (2013). Soft sheets of fibrillar bone from a fossil of the supraorbital horn of the dinosaur Triceratops horridus. Acta histochemica, 115(6), 603-608.

Asara, J. M., Schweitzer, M. H., Freimark, L. M., Phillips, M., y Cantley, L. C. (2007). Protein Sequences from Mastodon and Tyrannosaurus Rex Revealed by Mass Spectrometry. Science, 316(5822), 280.

Bertazzo, S., Maidment, S. C. R., Kallepitis, C., Fearn, S., Stevens, M. M., y Xie, H.-n. (2015). Fibres and cellular structures preserved in 75 -million-year-old dinosaur specimens. Nature Communications, 6, 7352. doi:10.1038/ncomms 8352

Briggs, D. E. G. (1991). Extraordinary Fossils. American Scientist, 79(2), 130-141.

Briggs, D. E. G., y McMahon, S. (2016). The role of experiments in investigating the taphonomy of exceptional preservation. Palaeontology, 59(1), 1-11. doi:10.1111/pala.12219

Briggs, D. E. G., Wilby, P. R., Perez-Moreno, B. P., Sanz, J. L., y Fregenal-Martínez, M. (1997). The mineralization of dinosaur soft tissue in the Lower Cretaceous of Las Hoyas, Spain. Journal of the Geological Society, 154(4), 587.

Buckley, M., Warwood, S., van Dongen, B., Kitchener, A. C., y Manning, P. L. (2017). A fossil protein chimera; difficulties in discriminating dinosaur peptide sequences from modern cross-contamination. Proceedings of the Royal Society B: Biological Sciences, 284(1855). doi:10.1098/rspb.2017.0544

Cadena, E. (2016). Microscopical and elemental FESEM and Phenom ProX-SEM-EDS analysis of osteocyte-and blood vessel-like microstructures obtained from fossil vertebrates of the Eocene 
Messel Pit, Germany. PeerJ, 4, e1618. https://doi.org/10.7717/peerj.1618

Cadena, E. A., y Schweitzer, M. H. (2012). Variation in osteocytes morphology vs bone type in turtle shell and their exceptional preservation from the Jurassic to the present. Bone, 51(3), 614-620.

Cartelle, C., y De luliis, G. (1995). Eremotherium laurillardi: The Panamerican late Pleistocene megatheriid sloth. Journal of Vertebrate Paleontology, 15(4), 830-841.

Chiappe, L. M., Coria, R. A., Dingus, L., Jackson, F., Chinsamy, A., y Fox, M. (1998). Sauropod dinosaur embryos from the Late Cretaceous of Patagonia. Nature, 396, 258. doi:10.1038/24370

Cleland, T. P., Schroeter, E. R., Feranec, R. S., y Vashishth, D. (2016). Peptide sequences from the first Castoroides ohioensis skull and the utility of old museum collections for palaeoproteomics. Proceedings of the Royal Society B: Biological Sciences, 283(1832). doi:10.1098/rspb.2016.0593

Cleland, T. P., Schroeter, E. R., Zamdborg, L., Zheng, W., Lee, J. E., Tran, J. C., . . Schweitzer, M. H. (2015). Mass Spectrometry and Antibody-Based Characterization of Blood Vessels from Brachylophosaurus canadensis. Journal of Proteome Research, 14(12), 5252-5262. doi:10.1021/acs.jproteome.5b00675

Webb, S., y C. Perrigo, S. (1984). Late Cenozoic vertebrates from Honduras and El Salvador. Journal of Vertebrate Paleontology, 4(2):237-254

de Ricqlès, A. J. (2007). Fifty years after Enlow and Brown's Comparative histological study of fossil and recent bone tissues (1956-1958): A review of Professor Donald H. Enlow's contribution to palaeohistology and comparative histology of bone. Comptes Rendus Palevol, 6(8), 591-601. doi:https://doi.org/10.1016/j.crpv.2007.09.010

Enlow, D., y Brown, S. (1956). A comparative histological study of fossil and recent bone tissues. Texas Journal of Science, 8, 405-443.

Erkens, R., y Hoorn, C. (2016). The Panama Isthmus, 'old', 'young' or both? A reply to O'Dea et al. 2016.

Fernández, E. (1983). Introducción a la Paleontología Vertebrada de Honduras. Yaxkin, 6(1 y 2), 31-47.

Franzen, J. L. (2017). Report on the Discovery of Fossil Mares with Preserved Uteroplacenta from the Eocene of Germany. Fossil Imprint, 73(1-2), 67-75.

Fritz-Gaertner, R. (1878). The Microscope as a Means of Examination of Rocks and Fossils. The American Naturalist, 12(1), 13-17.

Gallo, V., S Avilla, L., C.L. Pereira, R., y Absolon, B. (2013). Distributional patterns of herbivore megamammals during the Late Pleistocene of South America. Anais da Academia Brasileira de Ciências, 85(2), 533-546. https://dx.doi.org/10.1590/S0001-37652013000200005

Grimaldi, D. A., Bonwich, E., Delannoy, M., y Doberstein, S. (1994). Electron microscopic studies of mummified tissues in amber fossils. American Museum novitates; no. 3097.

Gross, W. (1934). Die Typen des mikroskopischen Knochenbaues bei fossilen Stegoccphalen und Reptilien. Zeitschrift für Anatomie und Entwicklungsgeschichte, 103(6), 731-764.

Hare, P. (1988). Organic geochemistry of bone and its relation to the survival of bone in the natural environment. In A. Behrensmeyer y A. Hill (Eds.), Fossils in the Making: Vertebrate Taphonomy and Paleoecology (pp. 208). Chicago y Londres: The University of Chicago Press.

Horne, G. S. (1994). A Mid-Cretaceous Ornithopod from Central Honduras. Journal of Vertebrate Paleontology, 14(1), 147-150.

IHCIT. (2017). Informe Preliminar Geológico del Afloramiento de Fósiles. Facultad de Ciencias, UNAH. Inoué, T., y Osatake, H. (1988). A New Drying Method of Biological Specimens for Scanning Electron Microscopy: The t-Butyl Alcohol Freeze-drying Method. Archives of Histology and Cytology, 51(1), 53-59. doi:10.1679/aohc.51.53 
Janko, M., Stark, R. W., y Zink, A. (2012). Preservation of 5300 year old red blood cells in the Iceman. Journal of the Royal Society Interface, 9(75), 2581-2590.

Jaramillo, C., Montes, C., Cardona, A., Silvestro, D., Antonelli, A., y Bacon, C. (2017). Comment (1) on "Formation of the Isthmus of Panama" by O'Dea et al (Vol. 3).

Kellner, A. W. A. (1996). Fossilized theropod soft tissue. Nature, 379, 32. doi:10.1038/379032a0

Lucas, S. G. (2014). Vertebrate paleontology in Central America: 30 years of progress. Revista Geologica de América Central, 139-155.

Lucas, S. G., y Alvarado, G. E. (2017). Vertebrate palaeontology in Central America: a narrative and analytical history. Geological Society, London, Special Publications, 442(1), 155.

Lucas, S. G., Bonta, M., Rogers, R., y Alvarado, G. E. (2011). The Tambla (Humuya) Gomphothere (Honduras): The first report of fossil vertebrates in Central America. Revista Geológica de América Central, (44), 141-151. Retrieved from http://www.scielo.sa.cr/scielo.php?script=sci_arttext\&pid=S0256-70242011000100009\&lng=en\&tIng=en.

Maat, G. J. R. (1993). Bone preservation, decay and its related conditions in ancient human bones from Kuwait. International Journal of Osteoarchaeology, 3(2), 77-86.

Manning, P. L., Morris, P. M., McMahon, A., Jones, E., Gize, A., Macquaker, J. H. S., . . Wogelius, R. A. (2009). Mineralized soft-tissue structure and chemistry in a mummified hadrosaur from the Hell Creek Formation, North Dakota (USA). Proceedings of the Royal Society B: Biological Sciences. 276(1672), 3429-3437.

Molnar, P. (2008). Closing of the Central American Seaway and the Ice Age: A critical review. Paleoceanography, 23(2).

Molnar, P. (2017). Comment (2) on "Formation of the Isthmus of Panama" by O'Dea et al (Vol. 3).

Montes, C., Cardona, A., Jaramillo, C., Pardo, A., Silva, J. C., Valencia, V., . . Niño, H. (2015). Middle Miocene closure of the Central American Seaway. Science, 348(6231), 226.

New York State Museum of Natural History. (1878). Annual Report on the New York State Museum of Natural History (Vol. 30): The Regents.

O'Reilly, S., Summons, R., Mayr, G., y Vinther, J. (2017). Preservation of uropygial gland lipids in a 48-million-year-old bird. Proceedings of the Royal Society B: Biological Sciences, 284(1865). doi:10.1098/rspb.2017.1050

Organ, C. L., Schweitzer, M. H., Zheng, W., Freimark, L. M., Cantley, L. C., y Asara, J. M. (2008). Molecular Phylogenetics of Mastodon and Tyrannosaurus rex. Science, 320(5875), 499.

Osborn, H. F. (1910). The age of mammals in Europe, Asia and North America: Macmillan.

Pappas, S. (2013). Controversial T. Rex Soft Tissue Find Finally Explained. In: Live Science, New York,.

Pawlicki, R. (1978). Morphological differentiation of the fossil dinosaur bone cells. Cells Tissues Organs, 100(4), 411-418.

Pawlicki, R., Korbel, A., y Kubiak, H. (1966). Cells, Collagen Fibrils and Vessels in Dinosaur Bone. Nature, 211, 655. doi:10.1038/211655a0

Plet, C., Grice, K., Pagès, A., Verrall, M., Coolen, M., Ruebsam, W., .. .y Schwark, L. (2017). Palaeobiology of red and white blood cell-like structures, collagen and cholesterol in an ichthyosaur bone. Scientific reports, 7(1), 13776.

Qiang, J., Currie, P. J., Norell, M. A., y Shu-An, J. (1998). Two feathered dinosaurs from northeastern China. Nature, 393, 753. doi:10.1038/31635

Rincón, A. (2004). Los mamíferos fósiles del Pleistoceno de la Cueva del Zumbador (Fa. 116), Estado Falcón, Venezuela (Vol. 37).Boletín de la Sociedad Venezolana de Espeleología, 37:18-26.

Sapper, K. (1905). Über gebirgsbau und boden des südlichen Mittelamerika: Justus Perthes. 
Sasso, C. D., y Signore, M. (1998). Exceptional soft-tissue preservation in a theropod dinosaur from Italy. Nature, 392, 383. doi:10.1038/32884

Schroeter, E. R., DeHart, C. J., Cleland, T. P., Zheng, W., Thomas, P. M., Kelleher, N. L., ... y Schweitzer, M. H. (2017). Expansion for the Brachylophosaurus canadensis collagen I sequence and additional evidence of the preservation of Cretaceous protein. Journal of proteome research, 16(2), 920-932.

Schweitzer, M. H., Moyer, A. E., y Zheng, W. (2016). Testing the hypothesis of biofilm as a source for soft tissue and cell-like structures preserved in dinosaur bone. PloS one, 11(2), e0150238.

Schweitzer, M. H., Watt, J. A., Avci, R., Knapp, L., Chiappe, L., Norell, M., y Marshall, M. (2002). Beta-keratin specific immunological reactivity in feather-like structures of the Cretaceous Alvarezsaurid, Shuvuuia deserti. Journal of Experimental Zoology, 285(2), 146-157. doi:10.1002/(SICl)1097-010X(19990815)285:2<146::AID-JEZ7>3.0.CO;2-A

Schweitzer, M. H., Wittmeyer, J. L., y Horner, J. R. (2007). Soft tissue and cellular preservation in vertebrate skeletal elements from the Cretaceous to the present. Proceedings of the Royal Society B: Biological Sciences, 274(1607), 183-197. doi:10.1098/rspb.2006.3705

Schweitzer, M. H., Wittmeyer, J. L., Horner, J. R., y Toporski, J. K. (2005). Soft-Tissue Vessels and Cellular Preservation in Tyrannosaurus rex. Science, 307(5717), 1952-1955. doi:10.1126/science. 1108397

Schweitzer, M. H., Zheng, W., Cleland, T. P., y Bern, M. (2013). Molecular analyses of dinosaur osteocytes support the presence of endogenous molecules. Bone, 52(1), 414-423.

Schweitzer, M. H., Zheng, W., Cleland, T. P., Goodwin, M. B., Boatman, E., Theil, E., . . Fakra, S. C. (2014). A role for iron and oxygen chemistry in preserving soft tissues, cells and molecules from deep time. Proceedings of the Royal Society B: Biological Sciences, 281(1775). doi:10.1098/rspb.2013.2741

Schweitzer, M. H., Zheng, W., Organ, C. L., Avci, R., Suo, Z., Freimark, L. M., . . Asara, J. M. (2009). Biomolecular Characterization and Protein Sequences of the Campanian Hadrosaur B. canadensis. Science, 324(5927), 626-631. doi:10.1126/science.1165069

Stone, R. (2013). Battle for the Americas. In: American Association for the Advancement of Science.

Varela, L., Tambusso, S., J. Patiño, S., Di Giacomo, M., y Fariña, R. (2017). Potential Distribution of Fossil Xenarthrans in South America during the Late Pleistocene: co-Occurrence and Provincialism. Journal of Mammalian Evolution, 1-12. doi: 10.1007/s10914-017-9406-9

Webb, S. D., y Perrigo, S. C. (1984). Late cenozoic vertebrates from Honduras and El Salvador. Journal of Vertebrate Paleontology, 4(2), 237-254.

Weishampel, D. B., Dodson, P., y Osmólska, H. (2004). The Dinosauria: University of California Press.

Xiao, S., y Knoll, A. H. (1999). Fossil preservation in the Neoproterozoic Doushantuo phosphorite Lagerstätte, South China. Lethaia, 32(3), 219-238. doi:10.1111/j.1502-3931.1999.tb00541.x

Xu, X., Wang, X.-L., y Wu, X.-C. (1999). A dromaeosaurid dinosaur with a filamentous integument from the Yixian Formation of China. Nature, 401, 262. doi:10.1038/45769

Zúniga, L. (2017a). Evidencia fósil de dinosaurios: un aporte a la historia de la paleontología en Centroamérica. Revista Ciencia Y Tecnología, 0(20), 29-49. doi:http://dx.doi.org/10.5377/rct.v0i20.5494

Zúniga, L. (2017b). Informe de Proyecto Paleontológico. In Fase 2: Escuela de Biología, Facultad de Ciencias, UNAH. 